\title{
Comparison between experiment and perturbation theory for solitons in Josephson junctions
}

\author{
Pedersen, Niels Falsig; Welner, D.
}

Published in:

Physical Review B

Link to article, DOI:

10.1103/PhysRevB.29.2551

Publication date:

1984

Document Version

Publisher's PDF, also known as Version of record

Link back to DTU Orbit

Citation (APA):

Pedersen, N. F., \& Welner, D. (1984). Comparison between experiment and perturbation theory for solitons in Josephson junctions. Physical Review B, 29(5), 2551-2557. https://doi.org/10.1103/PhysRevB.29.2551

\section{General rights}

Copyright and moral rights for the publications made accessible in the public portal are retained by the authors and/or other copyright owners and it is a condition of accessing publications that users recognise and abide by the legal requirements associated with these rights.

- Users may download and print one copy of any publication from the public portal for the purpose of private study or research.

- You may not further distribute the material or use it for any profit-making activity or commercial gain

- You may freely distribute the URL identifying the publication in the public portal

If you believe that this document breaches copyright please contact us providing details, and we will remove access to the work immediately and investigate your claim. 


\title{
Comparison between experiment and perturbation theory for solitons in Josephson junctions
}

\author{
N. F. Pedersen and D. Welner \\ Physics Laboratory I, The Technical University of Denmark, DK-2800 Lyngby, Denmark
}

(Received 19 May 1983)

\begin{abstract}
Experiments have been made on long inline and overlap Josephson junctions at various temperatures and current densities. The junctions had parameters such that the recently developed perturbation theory for soliton motion according to the modified sine-Gordon equation should be applicable. A comparison showed that this is the case, and the damping constant was derived as a function of the temperature. In addition, results were obtained for the soliton-antisoliton annihilation process. A fine structure in the zero-field steps at low temperatures is interpreted as being due to plasma oscillations in connection with soliton reflections at the boundaries.
\end{abstract}

\section{INTRODUCTION}

The topic of solitons in long Josephson junctions has attracted considerable interest in recent years. The aim of the present paper is to make a detailed comparison between experiments and the recently developed perturbation theory for soliton motion in the modified sineGordon equation. ${ }^{1}$ Previous comparisons ${ }^{2,3}$ are based on very time-consuming numerical computations and thus are restricted to a few parameter values. In the present work the junctions had normalized lengths larger than 25 and damping constants less than 0.1 , a parameter range ideal for a comparison with the analytic results of the perturbation theory. ${ }^{1,4,5}$ The comparison gives values of the damping constant that are confirmed by an independent calculation. Also, experimental results for the solitonantisoliton annihilation threshold are compared with predictions of the perturbation theory. Finally, a fine structure in the $I-V$ curves at low temperatures is interpreted as soliton interaction with plasma oscillations in contradiction to previous suggestions.

The paper is organized as follows. In Sec. II a short review of the perturbation theory for soliton motion in the modified sine-Gordon equation is given. Section III describes the samples investigated and shows some representative $I-V$ curves. Section IV deals with the soliton dynamics obtainable from the $I-V$ curves, Sec. V deals with soliton-antisoliton annihilation, and Sec. VI discusses the fine structure observed at low temperatures. The paper is concluded with a short summary.

\section{SUMMARY OF PERTURBATION RESULTS}

The long Josephson junction is assumed to be described by the modified sine-Gordon equation ${ }^{1}$

$$
-\phi_{x x}+\phi_{t t}+\alpha \phi_{t}+\sin \phi=\eta \text {. }
$$

Here $\phi(x, t)$ is the space- and time-dependent phase difference between the two superconducting films. The spatial variable $x$ is measured in units of the Josephson penetra- tion depth $\lambda_{J}=\left(\hbar / 2 \mu_{0} e d J\right)^{1 / 2}$, and time $t$ in units of the reciprocal plasma frequency $\omega_{0}^{-1}$, where $\omega_{0}=(2 e J / \hbar c)^{1 / 2}$. $J$ is the maximum pair current density, $d$ is the magnetic thickness of the barrier $\left(d=\lambda_{1}+\lambda_{2}+t_{0}\right)$, and $c$ is the capacitance per unit area. The parameter $\alpha$ is the damping constant, $\alpha=G(\hbar / 2 e J c)^{1 / 2}$, where $G$ is the shunt conductance per unit area. $\alpha=1 /\left(\beta_{c}\right)^{1 / 2}$, where $\beta_{c}$ is the usual McCumber parameter. For an overlap junction [inset in Fig. 2(a)] with uniform current distribution, $\eta=I_{\mathrm{dc}} / I_{0}^{\mathrm{ov}}$. Here $I_{\mathrm{dc}}$ is the bias current and $I_{0}^{\mathrm{ov}}=J W L$ is the critical current of a junction of length $L$ and width $W$. The perturbation theory requires $l=L / \lambda_{J} \gg 1$, $w=W / \lambda_{J} \ll 1$, and $\alpha<1$.

For the overlap geometry the perturbation theory 4,5 with boundary conditions $\phi_{x}(0, t)=\phi_{x}(l, t)=0$ leads to a steady-state soliton velocity $u_{\infty}$ (normalized to $\bar{c}=\lambda_{J} \omega_{0}$ ) given by

$$
u_{\infty}=\left[1+(4 \alpha / \pi \eta)^{2}\right]^{-1 / 2},
$$

and so-called zero-field steps occur in the $I-V$ curve at voltages (normalized to $\hbar \omega_{0} / 2 e$ ),

$$
v_{n}=n 2 \pi u_{\infty} / l \text {, }
$$

where $n$ is the number of steps.

For the inline case ${ }^{4,5}$ [geometry shown in inset in Fig. 2(b)] the maximum critical current is $I_{0}^{\text {in }}=4 \lambda_{J} W J$. The current is flowing in an antisymmetric way $[\eta=0$ in Eq. (1)] and enters only through the boundary conditions $\phi_{x}(0, t)=-\phi_{x}(l, t)=\kappa$, where $\kappa=I_{\mathrm{dc}} / 2 W \lambda_{J} J$. Even though the dynamics are quite different, zero-field steps occur here much the same way as in the overlap case. ${ }^{4,5}$ They are given by

$$
\begin{aligned}
& \kappa=\frac{I_{\mathrm{dc}}}{2 \lambda_{J} W J}=\frac{\sinh (\alpha l / 2)}{\left[\left(\frac{\tanh \left(\alpha l / 2 u_{\mathrm{av}}\right)}{\tanh (\alpha l / 2)}\right)^{2}-1\right]^{1 / 2},} \\
& v_{n}=n 2 \pi u_{\mathrm{av}} / l .
\end{aligned}
$$


Theoretical $I-V$ curves for inline and overlap junctions are shown in Refs. 4 and 5. For the same value of $I_{\mathrm{dc}}$ the voltage of the inline junction is smaller than that of the overlap junction, and the current range is more restricted. In the limit $\alpha l \ll 1$ the two cases become identical in an absolute current scale. ${ }^{4}$

\section{SAMPLES AND I-V CURVES}

The samples investigated were niobium-lead junctions made on glass substrates. Each substrate had both an inline and an overlap junction to ensure the same current density for the junctions to be compared. The dimensions of both inline and overlap junctions were approximately $L \times W=1000 \times 15 \mu \mathrm{m}^{2}$. The two sets of samples investigated in detail had $J \cong 270 \mathrm{~A} / \mathrm{cm}^{2}$ and $J \cong 570 \mathrm{~A} / \mathrm{cm}^{2}$ at $4.2 \mathrm{~K}$. This corresponds to normalized lengths $l$ of 35 and 50 , respectively, satisfying the condition $l \gg 1$ from perturbation theory. In calculating the current density $70 \%$ of the current increase at the gap, $I_{0}^{\prime}=0.7 \Delta I_{g}$ (Fig. 1), was used instead of the measured maximum supercurrent $I_{0}$ to eliminate effects of a spatial variation of the bias current. $^{6}$

Figure 1 shows the full $I-V$ curve of the overlap junction with $J=270 \mathrm{~A} / \mathrm{cm}^{2}$. Note that the $I-V$ curve shows the expected behavior; however, the critical current does not reach $70 \%$ of the current increase at the gap as for small junctions. The reason is the following: Since a superconducting ground plane was not used, the current in the $1000-\mu \mathrm{m}$-wide film leading to the junction [Fig. 2(a)] had a nonuniform current distribution with singularities at the edges. The bias current of the junction, $\eta(x)$, may then be approximated by a uniform current distribution with spikes at $x=0$ and $l$. As the phase $\phi$ can only be adjusted on a length scale of order $\lambda_{J}$ this will become a typical length scale for the spikes at the ends. When the current at the edges exceeds the critical current density the junction will switch prematurely as seen in Fig. 1. In a junction of the same geometry but with $J \cong 0.1 \mathrm{~A} / \mathrm{cm}^{2}$ and $\lambda_{J} \approx 1 \mathrm{~mm}, I_{0}$ was found to be $0.7 \Delta I_{g}$, in agreement with the discussion above. A model calculation of the current distribution in a superconducting film without a superconducting ground plane ${ }^{7}$ shows that a fraction of

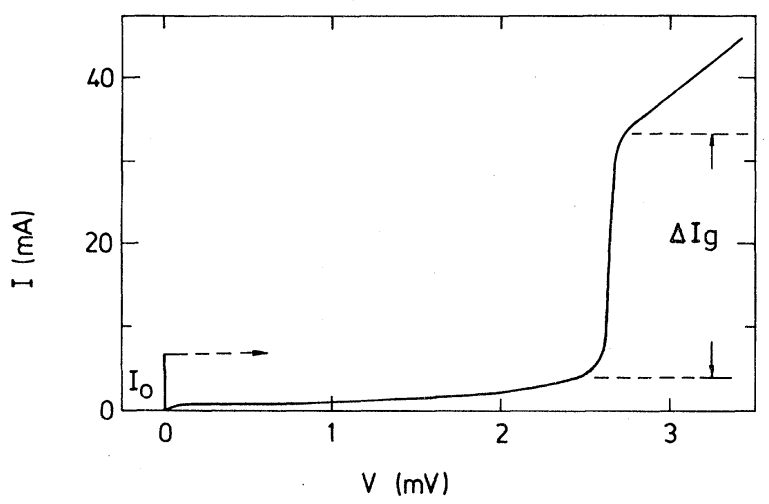

FIG. 1. $I-V$ curve of the overlap junction with $J=270 \mathrm{~A} / \mathrm{cm}^{2}$ $(T=4.2 \mathrm{~K})$.
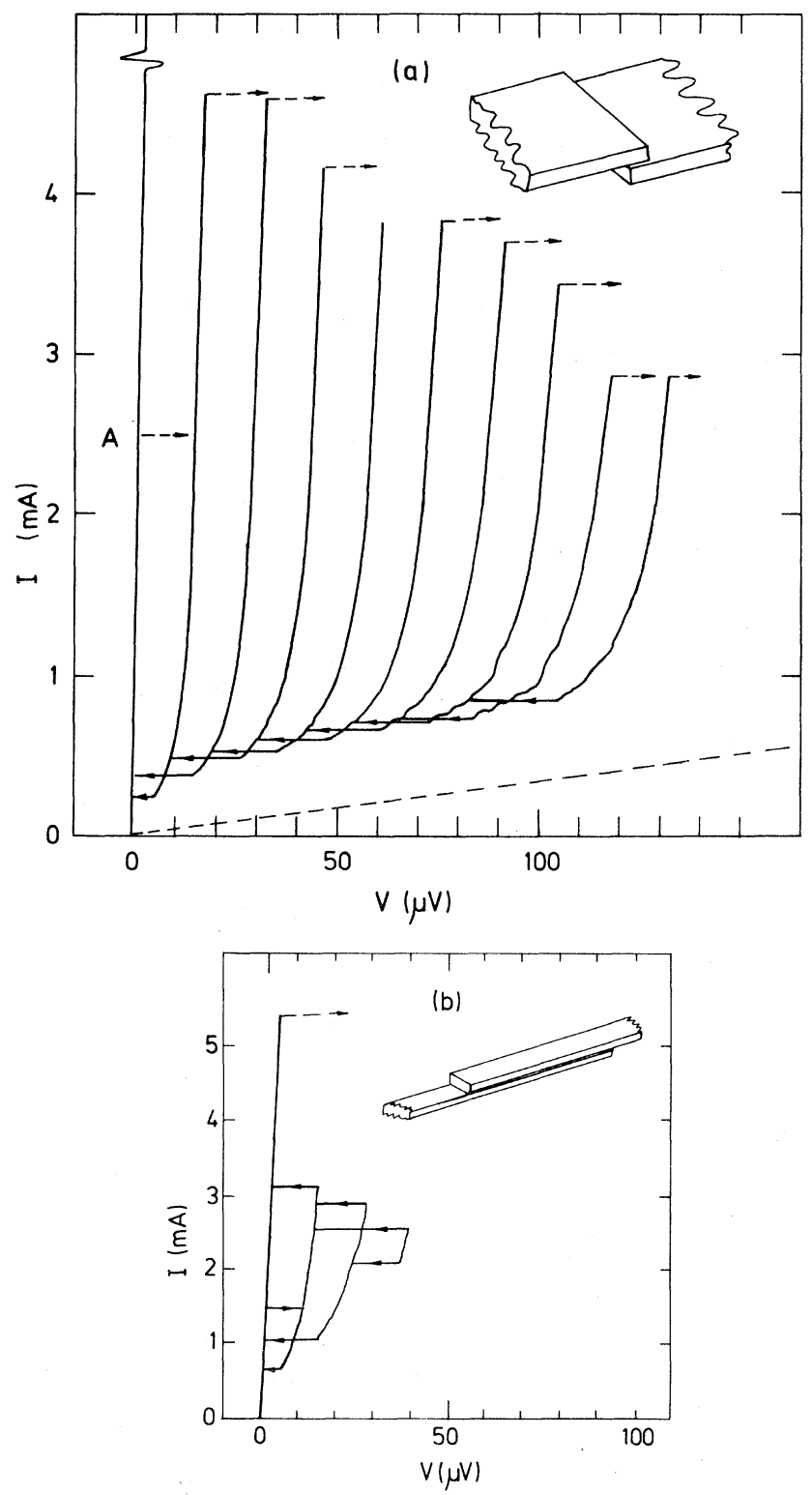

FIG. 2. Zero-field steps of (a) overlap junction and (b) inline junction: $J=270 \mathrm{~A} / \mathrm{cm}^{2}, T=4.2 \mathrm{~K}$. Insets show the overlap and inline geometries. Dashed curve is obtained with a large magnetic field. Supercurrent is slightly nonvertical due to resistance in bias leads.

$1-2 / \pi$ of the bias current flows in the current spikes at $x=0$ and $l$. From the reduction in the measured supercurrent one can estimate the normalized lengths of the junction. ${ }^{7}$ The results $l \simeq 30$ for the junction with $J=270$ $\mathrm{A} / \mathrm{cm}^{2}$ and $l \simeq 40$ for the junction with $J=570 \mathrm{~A} / \mathrm{cm}^{2}$ are in reasonable agreement with the estimates based on the current density. In the inline junction [Fig. 2(b)] the bias current is fed to the junction through a film of a width that is small compared with $\lambda_{J}$, and the effect discussed above is not expected to occur.

The current spikes at the edges of the overlap junction change the boundary conditions at $x=0$ and $l$, and make it in principle of a mixed overlap and inline character. ${ }^{8}$ However, with a fraction of $1-2 / \pi$ of the current flow- 


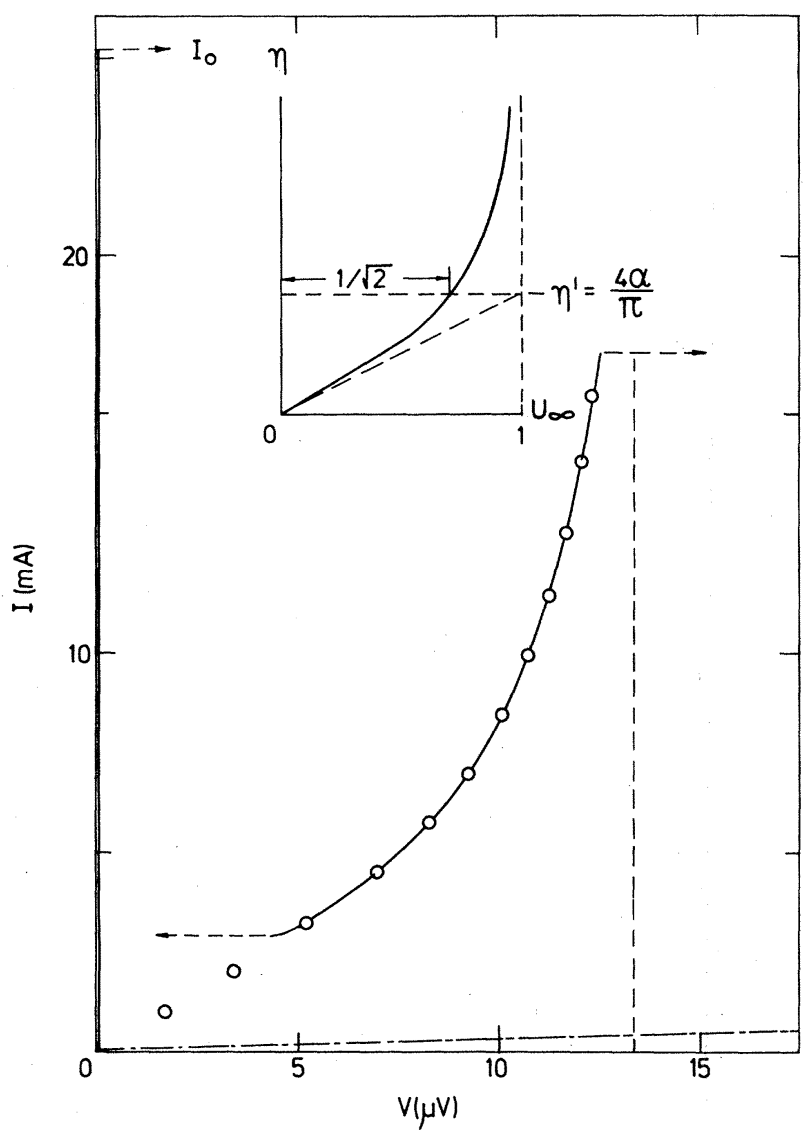

FIG. 3. Overlap junction, $J=570 \mathrm{~A} / \mathrm{cm}^{2}, T \simeq 5.2 \mathrm{~K}, n=1$ : Simple fit to Eq. (2). Inset shows the fitting procedure. The dashed-dotted curve corresponds to a large applied magnetic field.

ing in the spikes the $y$ parameter of Ref. 8 becomes $2 / \pi$ and the $I-V$ curve is very close to the pure overlap case. Thus, the main effect of neglecting the spikes is an incorrect normalization of $\eta$. A value of $\alpha$ obtained by a fit to Eq. (2) will be incorrect by a factor $I_{0}^{\prime} / I_{0}(\sim 3$ in Fig. 1).

Figure 2(a) shows in an expanded scale the zero-field steps near the origin for the junction in Fig. 1, and Fig. 2(b) shows the zero-field steps for the inline junction on the same substrate. The depression of the critical current in the inline junction, ${ }^{9}$ relative to $70 \%$ of the gap increase, gives an independent estimate of $l$ since $I_{0}^{\text {in }} / 0.7 \Delta I_{g}=4 \lambda_{J} / L$. The result for Fig. 2 (b) is $l \simeq 30$, in agreement with the previous estimates. Also shown in Fig. 2(a) is the $I-V$ curve when a magnetic field sufficiently large to quench the Josephson effect is applied. This $I$ $V$ curve is the subgap quasiparticle resistance at low voltages, $R_{\mathrm{QP}}$, and will become an important parameter in the next section.

\section{COMPARISON BETWEEN EXPERIMENT AND PERTURBATION THEORY}

Figure 3 shows for the overlap junction with $j \sim 570$ $\mathrm{A} / \mathrm{cm}^{2}$ at high temperatures a simple fit of the $n=1$ step to the perturbation result of Eqs. (2) and (3). The fitting is done in the following way. First the voltage of the asymptote is determined by inspection (this is often easiest to do with an applied magnetic field). The value of the current $I_{\mathrm{dc}}^{*}$ where the voltage has reached $1 / \sqrt{2}$ times its asymptotic value is normalized to the measured critical current $I_{0}$ (see inset in Fig. 3). Then the solid curve is calculated with

$$
\alpha^{\prime}=\frac{\pi}{4} \frac{I_{\mathrm{dc}}^{*}}{I_{0}} .
$$

As can be seen from Fig. 3 the fit is quite perfect and $\alpha$ can be determined very well. The spike effect is taken into account by normalizing the current to $0.7 \Delta I_{g}$ instead of $I_{0}$. Since $u_{\infty}$ depends only on the combination $\eta / \alpha$ this new normalization reduces the fitted values of $\alpha$ with the factor $0.7 \Delta I_{g} / I_{0}$. However, it leaves the fit in Fig. 3 unchanged. We found experimentally that the ratio $0.7 \Delta I_{g} / I_{0}$ is independent of temperature, consistent with the nature of the spike effect. For both of the overlap junctions the first zero-field step could be fitted to $u_{\infty}$, as shown in Fig. 3, as long as the temperature was not too low ( $T \gtrsim 4.2 \mathrm{~K}$ ). The fitted values of $\alpha$ decrease strongly with temperature. For lower temperatures the shape of the zero-field step changed qualitatively, and a simple one-parameter fit as in Fig. 3 was no longer possible. We suggest the reason is that $\alpha$ becomes so small that other damping mechanisms become important. In order to explore that possibility we have included surface-impedance damping in the model. This adds ${ }^{1,10}$ another term-

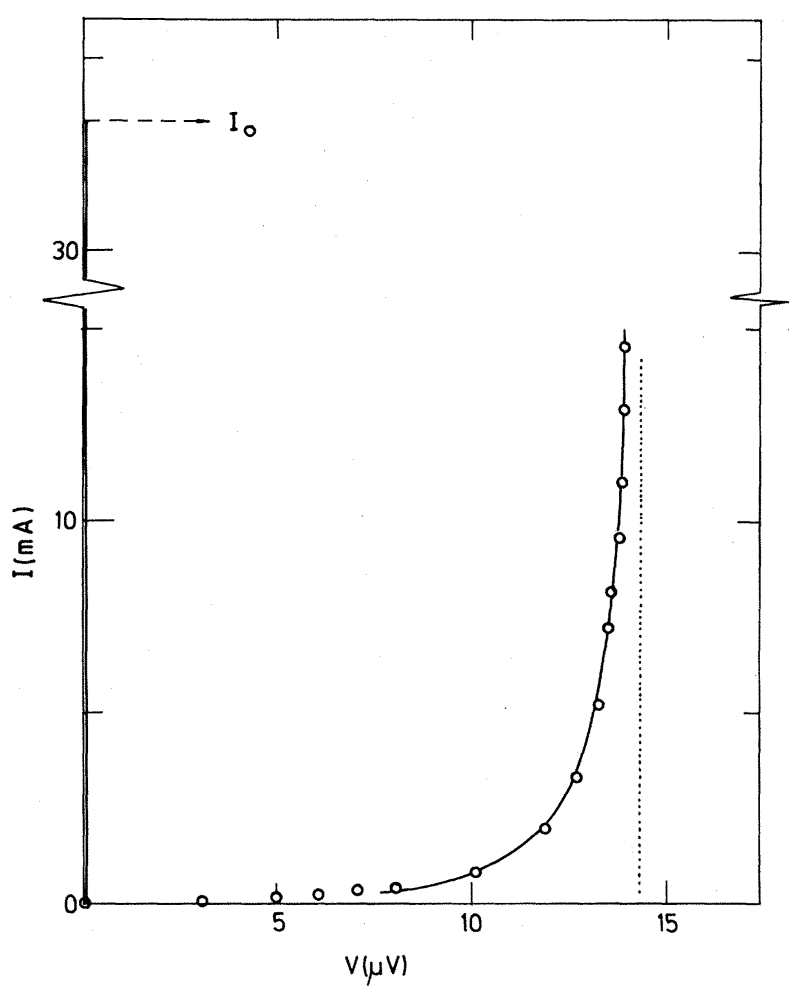

FIG. 4. Overlap junction, $J=570 \mathrm{~A} / \mathrm{cm}^{2}, T \simeq 2 \mathrm{~K}, n=1$ : Computer fit with $\alpha$ and $\beta$ at low temperatures. 


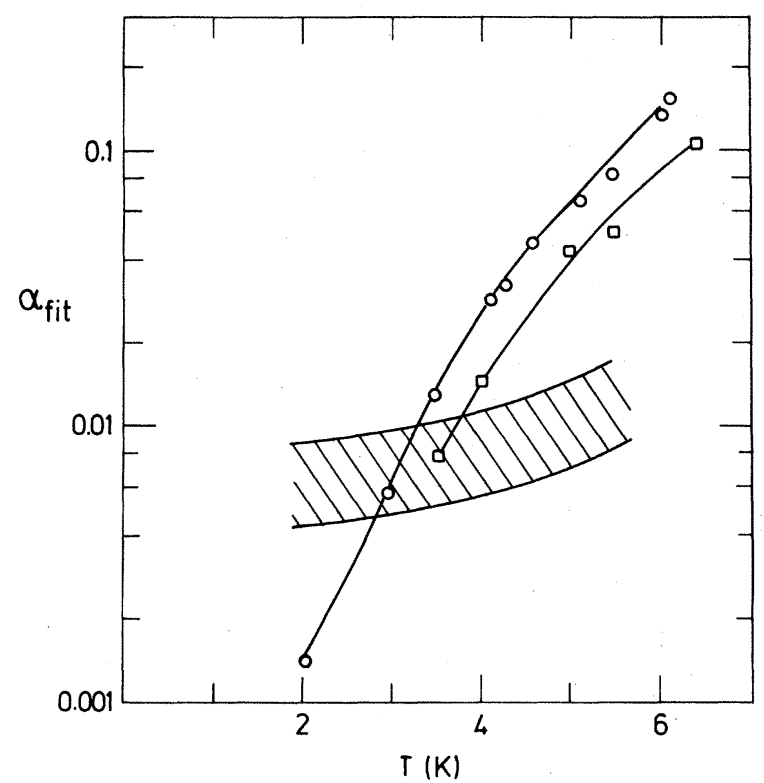

FIG. 5. Fitted values of $\alpha$. Circles $J=570 \mathrm{~A} / \mathrm{cm}^{2}$, squares $J=270 \mathrm{~A} / \mathrm{cm}^{2}$. Cross-hatched region gives the range of fitted $\beta$ 's. Smooth curves have been drawn through the data.

$\beta \phi_{x x t}$-in Eq. (1), and $u_{\infty}$ is determined from the following equation ${ }^{10}$ :

$$
\eta=\frac{4}{\pi} \frac{u_{\infty}}{\left(1-u_{\infty}\right)^{1 / 2}}\left(\alpha+\frac{\beta}{3\left(1-u_{\infty}^{2}\right)}\right) .
$$

With $\beta=0$ Eq. (6) reproduces Eq. (2). The fitting of $\alpha$ and $\beta$ in Eq. (6) to the experimental $I-V$ curves was done by a two-parameter fit on a desk computer, and a typical example is shown in Fig. 4. As can be seen the fit is quite satisfactory and values of $\alpha$ and $\beta$ can be derived.

The preceding discussion may be understood best by considering the results for $\alpha(T)$ as shown in Fig. 5. For both of the junctions $\alpha$ is a strongly varying function of temperature, spanning more than two decades. Note that the ratio between the $\alpha$ values of the two junctions is about 1.5 , consistent with the expectation from the definition of $\alpha$ that it varies as the square root of the current density. $\beta$ is in general small and the determination not very precise since it depends very critically on the value chosen for $\bar{c}$ (given by the asymptote). The general range of $\beta$ is shown as the cross-hatched region. The values of $\alpha$ and $\beta$ obtained at $4.2 \mathrm{~K}$ are in good agreement with the computer-fitted values obtained in Ref. 2 for similar types of junctions. For values of $\alpha$ such that $\alpha \gg \beta(T \geqslant 3.5-4$ $\mathrm{K})$. the effect of a small $\beta$ term does not introduce much uncertainty in the $\alpha$ determination. For small values of $\alpha$ $(T \lesssim 3 \mathrm{~K})$ the uncertainty in $\beta$ gives a similar uncertainty in $\alpha$. In principle, the low-temperature end of Fig. 5 could be improved by an improved measurement of $\bar{c}(T)$ and possibly by using a three-parameter fit with $\alpha, \beta$, and $\bar{c}$. This is beyond the scope of the present paper.

A natural question to ask is: What gives the strong temperature variation of $\alpha$ ? From Eq. (1), $\alpha=1 /\left(\beta_{c}\right)^{1 / 2}$, where $\beta_{c}=2 e I_{0} R^{2} C / \hbar$ is the McCumber parameter.

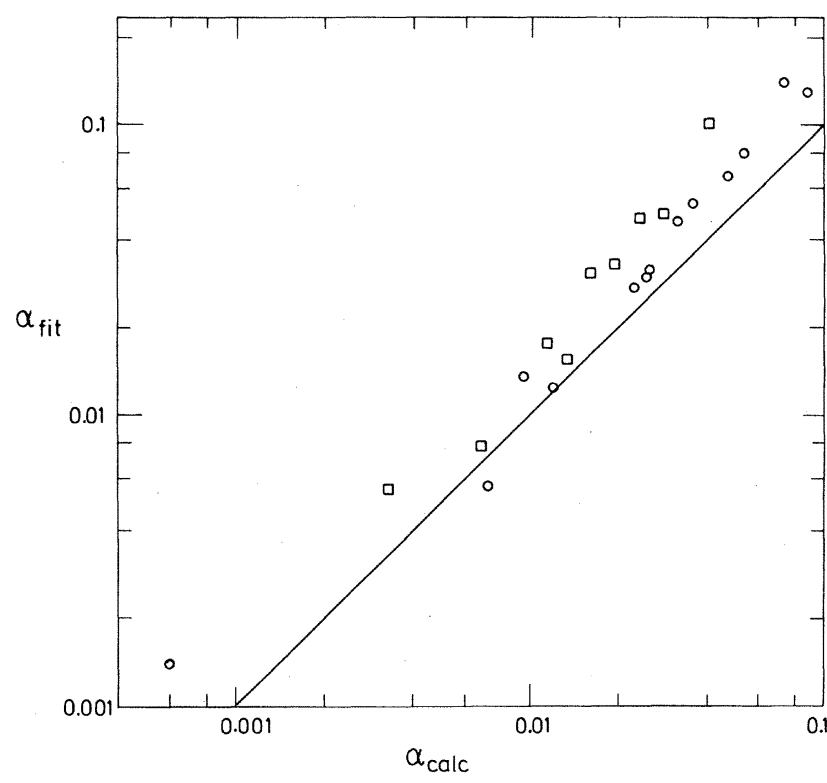

FIG. 6. Fitted values of $\alpha$ and $\alpha_{\text {fit }}$, plotted against calculated values of $\alpha$ and $\alpha_{\text {calc. }}$. Circles $J=570 \mathrm{~A} / \mathrm{cm}^{2}$, squares $J=270$ $\mathrm{A} / \mathrm{cm}^{2}$.

Since the capacitance does not change with temperature, and since $I_{0}$ varies only about a factor of 2 in our experiment, these observations cannot explain a variation of $\alpha$ over more than two decades. For the resistance $R$ the normal-state resistance $R_{N}$ is often used in estimates of the McCumber parameter $\beta_{c} . R_{N}$, however, does not vary with temperature. We found that the resistance $R_{\mathrm{QP}}$ obtained at low voltages when a large magnetic field is applied accounts very well for the observed temperature variation of $\alpha$. The resistance $R_{\mathrm{QP}}$ was introduced in connection with Fig. 2(a), and its origin is tunneling quasiparticles below the gap. The very strong temperature dependence of $R_{\mathrm{QP}}$ reflects the fact that we are working at the lower side of the $\Delta_{1}-\Delta_{2}$ singularity in a junction with two different superconductors.

Figure 6 shows a diagram of the fitted values $\alpha_{\text {fit }}$ versus the calculated values $\alpha_{\text {calc }}$. In all data $I_{0}^{\prime}=0.7 \Delta I_{g}$ was used, and in $\alpha_{\text {calc }}, G=1 / R_{\mathrm{QP}}$ was inserted, as discussed above. The capacitance $C$ was determined from the measurement of $\bar{c}$ and the dimensions of the junction. ${ }^{11}$ For the $570-\mathrm{A} / \mathrm{cm}^{2}$ junction we find $C=2.33 \mathrm{nF}$ (13.1 $\left.\mu \mathrm{F} / \mathrm{cm}^{2}\right)$, and for the $270-\mathrm{A} / \mathrm{cm}^{2}$ junction we find $C=1.79 \mathrm{nF}\left(11.9 \mu \mathrm{F} / \mathrm{cm}^{2}\right)$. Since a realistic uncertainty on the capacitances ${ }^{11}$ is much larger than this difference we have chosen to use $C=2.0 \mathrm{nF}$ for both junctions. Allowing for the various uncertainties we conclude from Fig. 6 that our determination of $\alpha$ is correct, and that it can be determined from a knowledge of $I_{0}$ (or $\Delta I_{g}$ ), $C$, and $R_{\mathrm{QP}}$.

As to the results for $\beta$ a critical remark is appropriate: Although we found it possible to obtain a reasonable fit at low temperatures by introducing this term, and although the order of magnitude was correct compared to other similar work, our fitting procedure does not exclude the possibility of other corrections to replace or supplement the $\beta$ term. Such corrections could, for example, be (i) 


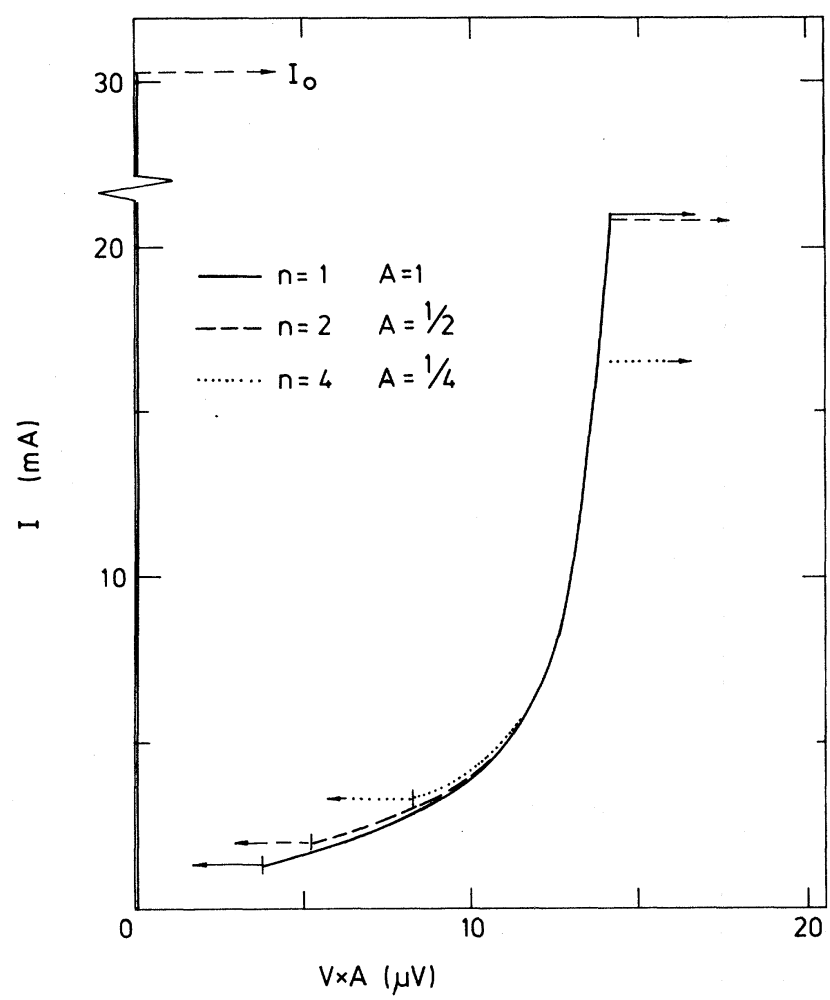

FIG. 7. Overlap junction, $J=570 \mathrm{~A} / \mathrm{cm}^{2}, T=4.2 \mathrm{~K}$. Zerofield steps $n=1$ (solid curve), $n=2$ (dashed curve), and $n=4$ (dotted curve) recorded with relative amplifications of $1, \frac{1}{2}$, and $\frac{1}{4}$.

nonlinearities in $R_{\mathrm{QP}}$ [an additional term $\delta \phi_{t}^{3}$ in Eq. (1) will lead to a correction similar to that for $\beta$ in Eq. (6)], (ii) deviations from Eq. (2) occurring when $\eta / \alpha$ becomes large, ${ }^{12}$ or (iii) a $\cos \phi$ damping term possibly being included in Eq. (1). At the present stage the derivation of $\beta$ from experiment is not sufficiently precise to give a definite answer to such questions.

The perturbation theory predicts the voltage of higherorder steps to scale with $n$. Figure 7 shows the experimental results. Here the steps numbered 1, 2, and 4 are recorded with relative voltage amplifications of 4,2 , and 1 , respectively. Note that at the bottom of the steps, deviations from scaling occur. This is not contained in the perturbation theory and has its origin in a time delay connected with either fluxon-antifluxon collisions or collisions with the boundaries. At lower temperatures (smaller $\alpha$ ) we found more perfect scaling.

Figure 8 shows the $n=1$ step of an inline and an overlap junction with equal dimensions and current density $\left(J \sim 270 \mathrm{~A} / \mathrm{cm}^{2}\right)$. For this temperature $\alpha \simeq 0.017$ and $l \simeq 35$ and we obtain $\alpha l \simeq 0.6$. The perturbation calculation $^{4}$ predicts for $\alpha l \rightarrow 0$ that in absolute current and voltage scales the two $I-V$ curves should be identical, except that the current range is more restricted for the inline junction. For $\alpha l \lesssim 1$ the inline $I-V$ curve $^{5}$ should be slightly on the inside of the overlap junction and should return to zero voltage at a finite value of the bias current. Except for the switching at the bottom of the zero-field

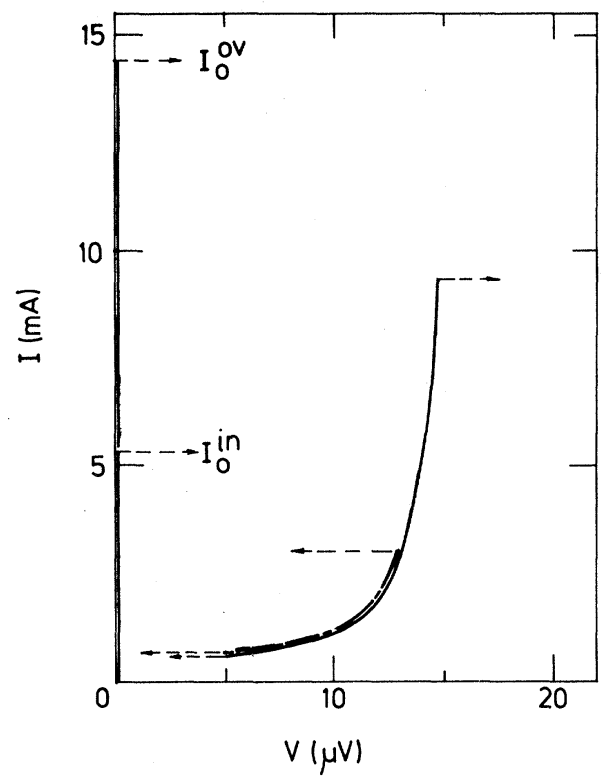

FIG. 8. $J=270 \mathrm{~A} / \mathrm{cm}^{2}, T=4.2 \mathrm{~K} . n=1$ zero-field step of overlap (solid curve) and inline (dashed-dotted curve) junctions.

steps, these qualitative features are observed in Fig. 8. A fit to the inline junction $I-V$ curve using Eqs. (4) and (5) gives a value of $\alpha l \cong 0.6$, in agreement with the previous estimate.

\section{SOLITON-ANTISOLITON ANNIHILATION AND STORAGE}

As is evident from the $I-V$ curve deviations from the simple model of moving solitons occur at the bottom of the zero-field steps where the $I-V$ curves switch. For the overlap junctions two solitons disappear simultaneously, i.e., as the current is decreased the junction switches through decreasing even steps or odd steps depending on the initial condition [Fig. 2(a)]. This is equivalent to a soliton-antisoliton annihilation process. For the $n=1$ step a special phenomenon was observed in both of the overlap junctions. If the junction switches to zero voltage from the bottom of the $n=1$ step the full critical current is not obtained on a subsequent current increase. The junction switches back to the $n=1$ step at the point $A$ in Fig. 2(a), and the procedure may be repeated. We interpret this as soliton storage at the end of the junction in the following way. Owing to the current spikes at the ends, as discussed in Sec. II, the boundary condition for the soliton at $x=0$ is $\phi_{x}(0, t)=\sigma$. Here $\sigma$ is determined by the amplitude and width $\left(\approx \lambda_{J}\right)$ of the current spike. The soliton may become trapped at the boundary when its value of $\phi_{x}$ is equal to $\sigma$. For $\sigma=0$ (no current spikes) the soliton cannot become stationary with a finite value of the bias, but will always move with the power balance velocity $u_{\infty}$ determined by Eq. (2). The stored soliton can be released only by decreasing the bias current through zero to negative values or by switching to finite voltages from the top of the $n=1$ step. We note here that this effect might be 


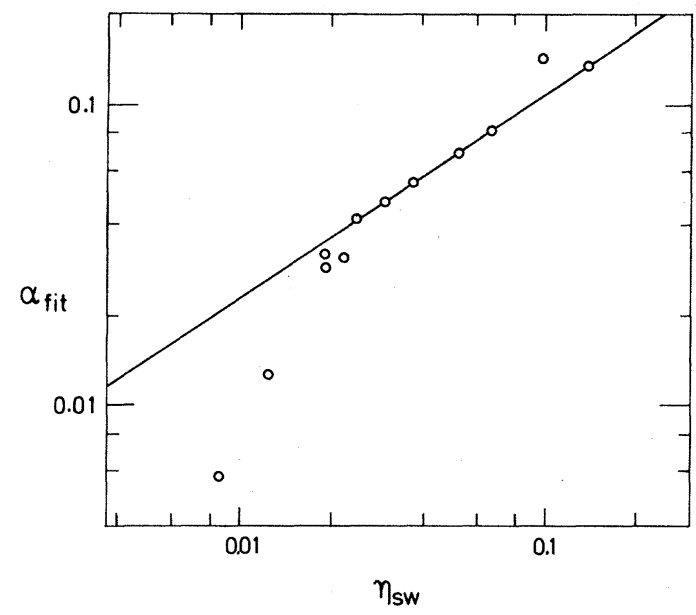

FIG. 9. Overlap junction, $J=570 \mathrm{~A} / \mathrm{cm}^{2} \cdot \eta_{\text {sw }}$ corresponding to lower threshold of $n=2$ step as a function of $\alpha_{\mathrm{fit}}$. Solid line: soliton-antisoliton annihilation curve after Ref. 1.

employed in a superconducting information storage system.

For the $n=2$ step no such phenomena was observed. Here, however, we expect the lower limit of soliton motion to be determined by a soliton-antisoliton annihilation process in the center of the line. For the infinite line this process was investigated within the framework of the perturbed sine-Gordon equation by McLaughlin and Scott. ${ }^{1}$ Since our line has a normalized length $l$ of about 50, a comparison seems justified. Figure 9 shows the normalized values of the bias current $\eta_{\mathrm{sw}}$ for the lower threshold of the $n=2$ step, together with the corresponding values of $\alpha$ obtained earlier. In the same diagram is also shown the curve for the soliton-antisoliton annihilation process on the infinite line as derived by McLaughlin and Scott. ${ }^{1}$ We note that there is agreement even beyond expectation taking the uncertainty of $\alpha$ into account. However, a pronounced deviation occurs for the lower values of $\alpha$. This is not unexpected, since from Fig. 5 this is the range where the surface-impedance damping $\beta$ becomes more important than $\alpha$, and a $\beta$ contribution was not included in the theoretical calculation. For the higher-order steps we cannot directly explain the increased lower threshold for soliton propagation which is observed experimentally [Fig. 2(a)]. We note, though, that as the line length per soliton becomes smaller on the higher-order steps, the effects of the finite size become important. For the lossless case $(\alpha=0)$ the lower stability limit for a finite-size junction was calculated in Ref. 13. From those results one may calculate a minimum soliton velocity ${ }^{14} u_{\min }$ possible in a junction of a given length. Qualitatively, this variation of $u_{\min }$ with $l$ may account for the increased lower threshold observed in Fig. 2(a); however, a more detailed comparison will require a model with a finite $\alpha$.

For the inline junction [Fig. 2(b)] the fluxon storage process on the $n=1$ step was also observed. Here the main qualitative difference from the overlap case is that the upper threshold of a step may imply switching to a lower step, i.e., losing a single soliton in the reflection pro-

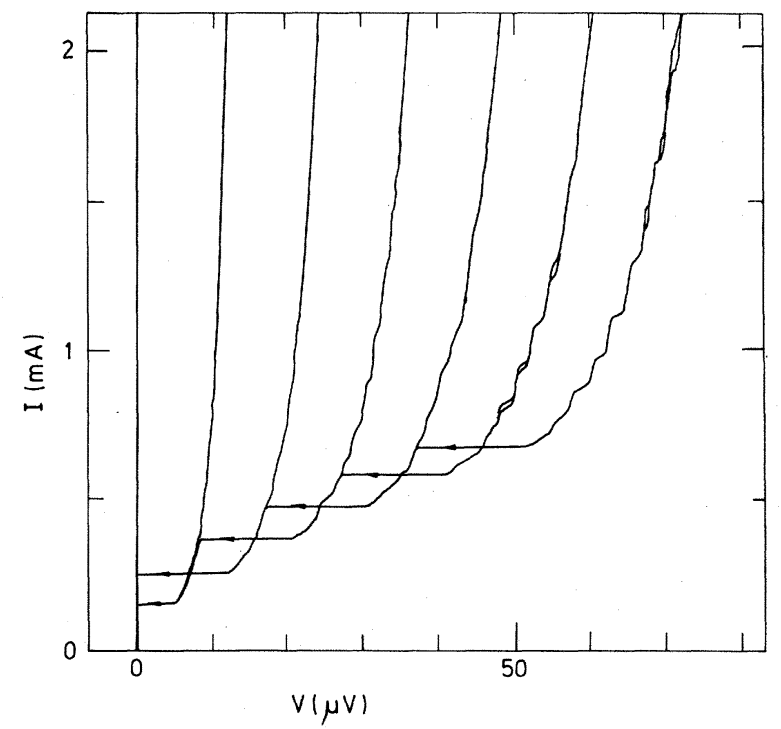

FIG. 10. Overlap junction, $J=570 \mathrm{~A} / \mathrm{cm}^{2}, T \simeq 2 \mathrm{~K}$. Fine structure of zero-field steps.

cess. The details were, however, temperature dependent $(\sim \alpha$ dependent). At $T \cong 3 \mathrm{~K}$ the same junction as in Fig. 2(b) showed four zero-field steps with switching characteristics exactly the same as those for the overlap junction in Fig. 2(b). Although in general only little is known of the detailed behavior of the reflection process at the boundary, we note that Fig. 8 in Ref. 15 may be used as an approximation to the inline junction with low damping. ${ }^{16} \mathrm{~A}$ meaningful comparison, however, would require more detailed calculations than those of Ref. 15.

\section{FINE STRUCTURE AT LOW TEMPERATURES}

Finally, it should be mentioned that at low temperatures a very regular fine structure is observed in the zero-field steps, as shown in Fig. 10. The voltage spacing between the fine structure steps scales with number $n$ of the zerofield step. A previous observation of such fine structure ${ }^{17}$ was interpreted as a fractional number cavity-mode structure at voltages $v=(p / q) \pi(\bar{c} / L)$ with $p$ and $q$ integer numbers. Our results may also be interpreted that way, and quite reasonable (low) numbers for $p$ and $q$ result.

However, such an explanation is not fully satisfactory since the dispersion relation for small oscillations (in normalized units) is $\omega^{2}=k^{2}+1$. Since $l \gg 1$, we obtain $k \simeq 0$ and $\omega \simeq 1$, and plasma oscillations-not cavity oscillations-are to be expected. Indeed, in numerical simulations, radiation in the form of plasma oscillations often occur in connection with the reflection of a soliton at the boundary. If $\alpha l \ll 1$, as is the case here at low temperatures $(\alpha l \simeq 0.04$ in Fig. 10), those plasma oscillations may persist for a long time. From the known current density and capacitance, the plasma frequency in Fig. 10 is estimated to about $60 \mathrm{GHz}$. Since the soliton frequency is of an order of a few gigahertz, the interaction between the soliton and the plasma wave is the following. As the soliton goes back and forth on the line, the whole line oscil- 


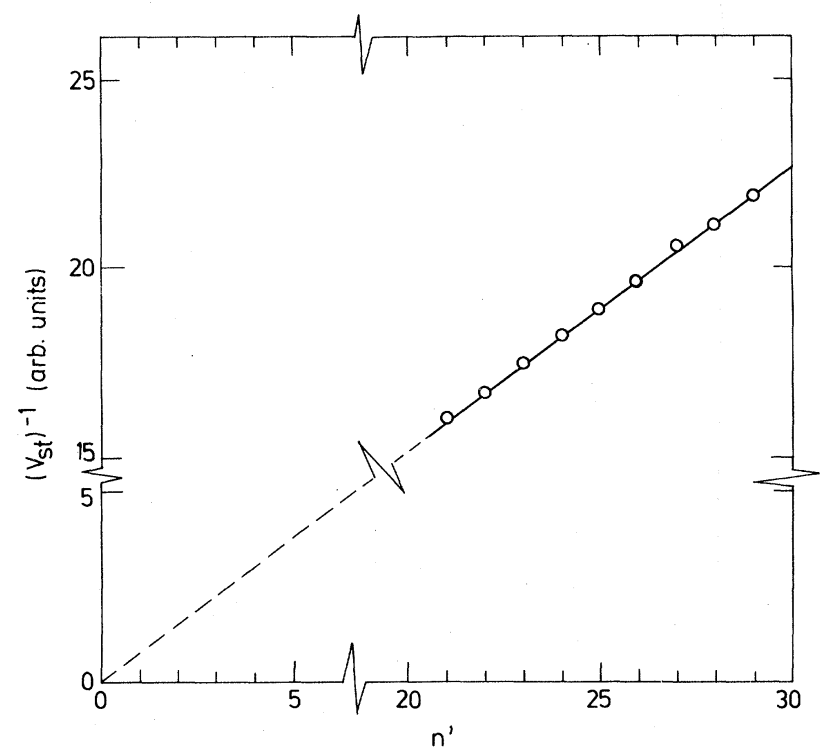

FIG. 11. Overlap junction, $J=570 \mathrm{~A} / \mathrm{cm}^{2}, T \simeq 2 \mathrm{~K} .\left(V_{\mathrm{st}}\right)^{-1}$ vs arbitrary numbering. Note the broken axes to determine $n^{\prime}$.

lates many times at the plasma frequency. An interference requires $n^{\prime} \omega_{\text {sol }}=\omega_{0}$, i.e., structure should be observed at voltages $V_{\text {st }}$ given by $n^{\prime} V_{\text {st }}=n 2 \hbar \omega_{0} / 2 e$, where $n^{\prime}$ is a number to be determined, and $n$ is the number of the zero-field step. In Fig. 11 consecutive values of $1 / V_{\text {st }}$ from Fig. 10 are plotted versus an arbitrary numbering. From the intersection of the line through the points with the $n^{\prime}$ axis we obtain the $n^{\prime}$ values $\left(n^{\prime} \sim 21-29\right)$ and find $f_{0} \simeq 65 \mathrm{GHz}$ in reasonable agreement with the estimated plasma frequency of $60 \mathrm{GHz}$. A close examination of the data published in Ref. 17 shows that an interpretation in terms of plasma oscillations instead of cavity oscillations is very well possible. It gives a plasma frequency of order $70 \mathrm{GHz}$, which is very reasonable for the stated current density.

As a conclusion of this section we note that both our data and the data in Ref. 17 may be interpreted in terms of either fractional cavity modes or plasma oscillations. Applying a magnetic field might possibly allow a distinction. Unfortunately, little is known of the magnetic field dependence of either type of oscillation. However, both experiments correspond to the strongly nonlinear limit, and the dispersion relation requires small amplitude oscillations to be plasma oscillations. Accepting that, the fine-structure oscillations give an independent method of determining the plasma frequency.

\section{SUMMARY}

The present work has shown that for long junctions with low damping the zero-field steps for both inline and overlap junctions may be compared with the simple results of the perturbation theory, and the damping constant may be derived. The value of the damping constant is determined by the quasiparticle resistance at low voltage, obtainable by applying a large magnetic field. For the niobium-lead junctions investigated here the damping constant is strongly temperature dependent, and at low temperatures the surface impedance should be taken into account. It was observed that owing to current spikes at the ends of the junction a soliton could become trapped there. On the second zero-field step the soliton-antisoliton annihilation threshold was found in agreement with predictions of the perturbation theory. Finally, the fine structure at low temperatures was identified as being due to plasma oscillations.

\section{ACKNOWLEDGMENTS}

The authors wish to acknowledge B. Dueholm for the sample fabrication. Stimulating discussions and numerous contributions from M. R. Samuelsen were very important for this work. The financial support of the Danish Natural Sciences Research Council is gratefully acknowledged.
${ }^{1}$ D. W. McLaughlin and A. C. Scott, Phys. Rev. A 18,1652 (1978).

2P. S. Lomdahl, O. H. Soerensen, and P. L. Christiansen, Phys. Rev. B 25, 5737 (1982).

${ }^{3}$ S. N. Erné, A. Ferrigno, T. Finnegan, and R. Vaglio, Physica 108B, 1301 (1981).

${ }^{4}$ O. A. Levring, N. F. Pedersen, and M. R. Samuelsen, Appl. Phys. Lett. 40, 846 (1982).

${ }^{5}$ O. A. Levring, N. F. Pedersen, and M. R. Samuelsen, J. Appl. Phys. 54, 987 (1983).

${ }^{6}$ The reduction factor of 0.7 is due to strong-coupling effects in the niobium-lead junctions. In small niobium-lead junctions we measure $I_{0} \simeq 0.7 \Delta I_{g}$.

${ }^{7}$ M. R. Samuelsen (private communication).

${ }^{8}$ O. H. Olsen and M. R. Samuelsen, J. Appl. Phys. (in press); B. Dueholm, E. Joergensen, O. A. Levring, R. Monaco, J. Mygind, N. F. Pedersen, and M. R. Samuelsen, IEEE Trans. Magn. MAG-19 (3) 1196 (1983).

${ }^{9}$ The spike effect from the overlap junction is not expected to occur in the inline junction.

${ }^{10}$ W. J. Johnson, Ph.D. thesis, University of Wisconsin, 1968 (unpublished).

${ }^{11}$ R. F. Broom, J. Appl. Phys. 47, 5432 (1976).

${ }^{12}$ M. Büttiker and H. Thomas, Phys. Lett. 77A, 372 (1980); M. Büttiker and R. Landauer, in Nonlinear Phenomena at Phase Transitions and Instabilities, edited by T. Riste (Plenum, New York, in press).

${ }^{13}$ G. Costabile, R. D. Parmentier, B. Savo, D. W. McLaughlin, and A. C. Scott, Appl. Phys. Lett. $\underline{32}, 587$ (1978).

${ }^{14}$ R. D. Parmentier (private communication).

${ }^{15}$ O. H. Olsen and M. R. Samuelsen, J. Appl. Phys. 52, 6247 (1981).

${ }^{16}$ In Fig. 8 of Ref. 15, $\beta$ contains the bias current and for $u$ should be substituted $u_{\mathrm{av}}-\alpha l / 2$, taking the velocity decay along the line into account.

${ }^{17}$ M. R. Scheuermann, T. V. Rajeerakumar, J.-J. Chang, and J. T. Chen, Physica 107B, 543 (1981). 